\title{
Metastatic ductal carcinoma of the prostate: a rare variant responding to a common treatment
}

\author{
Aalok Kumar, BESc, MD; ${ }^{*}$ Som Dave Mukherjee, MD, MSc, FRCPC ${ }^{\dagger}$
}

\begin{abstract}
Ductal carcinoma of the prostate is a rare histologic subtype of prostate carcinoma. It represents $0.4 \%$ to $0.8 \%$ of all prostate cancers and is associated with a poor prognosis. Given the paucity of cases reported in the literature on ductal prostate carcinoma, little is known about how this cancer responds to cytotoxic chemotherapy. We report the case of a 56-year-old male who presented to our clinic with hemoptysis, cough and hematuria and was found to have metastatic ductal carcinoma of the prostate. He was initiated on docetaxel chemotherapy, which has been previously shown to improve overall survival in patients with metastatic prostate adenocarcinoma, but has never been studied in this less common subtype of prostate cancer. To the best of our knowledge, the following is the first reported case of a patient with metastatic ductal carcinoma of the prostate responding to docetaxel chemotherapy.
\end{abstract}

Can Urol Assoc J 2010;4(2):E50-E54

\section{Introduction}

Ductal carcinoma of the prostate is a relatively rare subtype of prostate cancer. Very little literature exists on the natural history of this particular histological subtype; however in general, the prognosis for these patients is worse than those with typical adenocarcinoma of the prostate. Since the introduction of docetaxel into the management of patients with metastatic hormone refractory prostate cancer in 2004, there have been no published reports of patients with ductal carcinoma of the prostate responding to systemic chemotherapy. We report the case of a patient with this rare subtype of prostate cancer who was treated with docetaxel chemotherapy.

\section{Case presentation}

A 56-year-old man with a prior history of prostate cancer presented to his primary care physician with a 1-month history of cough, hemoptysis and shortness of breath on exertion. He underwent a computed tomography (CT) scan of the chest, abdomen and pelvis, and was found to have multiple lung metastases.

Ten years earlier, this gentleman presented with symptoms of gross hematuria and urinary hesitancy. He subsequently underwent a cystoscopy and biopsy of the prostate which led to a pathological diagnosis of ductal carcinoma of the prostate with endometrioid features (Fig. 1). His serum prostate-specific antigen (PSA) level at that time measured $3 \mu \mathrm{g} / \mathrm{L}$. A staging CT scan revealed no evidence of metastatic disease and a radical prostatectomy with pelvic lymph node dissection was performed. Final pathology confirmed this to be an invasive ductal carcinoma of the prostate with endometrioid features and a Gleason score of 8 . Positive resection margins were also identified and the patient received 4500 cGy of postoperative radiation to the pelvis in 25 fractions followed by an additional boost of 2000 cGy to the prostatic bed. The patient was then initiated on adjuvant gonadotropin-releasing hormone $(\mathrm{GnRH})$ agonist hormonal therapy and his serum PSA became undetectable at less than $0.03 \mu \mathrm{g} / \mathrm{L}$.

Four years following the completion of radiation, he developed hematuria and symptoms of urinary obstruction. Cystoscopy and bladder biopsies at that time confirmed recurrent ductal adenocarcinoma of the prostate and his serum PSA increased from less than 0.03 to $0.31 \mu \mathrm{g} / \mathrm{L}$. He underwent urinary diversion with an ileo-conduit and his PSA continued to increase (Table 1). The patient also developed cough, shortness of breath on exertion and hemoptysis, and was referred to medical oncology for a discussion of further treatment options. A bone scan was also performed and revealed no evidence of bony metastases. A staging CT scan of the chest, abdomen and pelvis revealed multiple nodules in both lungs measuring up to $2 \mathrm{~cm}$ in maximum diameter (Fig. 2, sections $A$ and $B$ ), but no other visceral metastases were found.

\section{Management}

After discussing options with the patient, we made the clinical decision to forego biopsy of the lung metastases for 


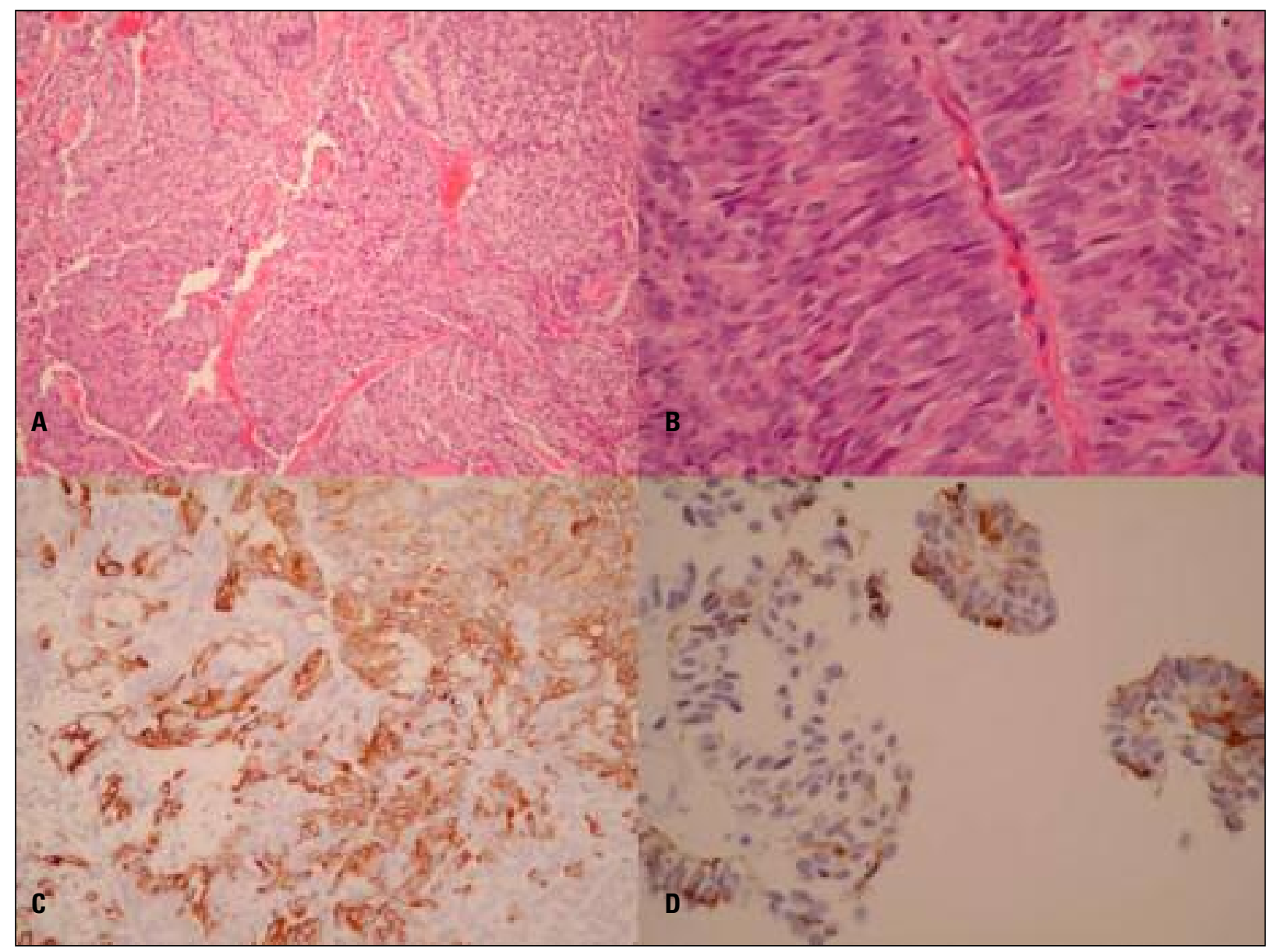

Fig. 1. Histopathology of ductal carcinoma of the prostate. (A) Cribriform pattern seen in ductal carcinoma. (B) Pleomorphic cells of ductal carcinoma. (C) Immunohistochemical staining positive for CK7. (D) Immunohistochemical staining positive for CK20.

the following reasons. (1) Histologic confirmation of locally recurrent ductal carcinoma of the prostate with endometrioid features had already been obtained with cystoscopy and several bladder biopsies. (2) The metastatic lung nodules were identified on CT scan at the same time as when the local recurrence of ductal carcinoma of the prostate was noted. (3) A review of the literature at that time revealed that visceral (lung and liver) metastases were relatively common in ductal prostate cancer compared to usual prostate adenocarcinoma ${ }^{1,2}$ and that PSA values were often normal in these patients, even in the setting of metastatic disease. ${ }^{1}$ (4) The patient had no past medical history of other primary malignancies. (5) Staging CT chest, abdomen and pelvis did not reveal any other abnormalities to suggest another primary site of disease. Based on these 5 reasons, the lung lesions identified on CT were deemed to be highly consistent with metastatic ductal carcinoma of the prostate. Given that the patient was symptomatic, he was initiated on intravenous docetaxel $75 \mathrm{mg} / \mathrm{m}^{2}$ every 3 weeks in combination with oral prednisone $5 \mathrm{mg}$ twice daily.

Following 4 cycles of docetaxel, many of the pulmonary nodules had decreased in size and his symptoms of hemoptysis, shortness of breath and cough improved significantly. In addition, his serum PSA decreased from 1.6 to less than $0.03 \mu \mathrm{g} / \mathrm{L}$. Although the serum PSA reached a maximum of only 1.6 prior to treatment, the decrease in size of the pulmonary nodules following chemotherapy occurred at the same time as the decrease in PSA to undetectable levels. The patient received an additional 6 months of chemotherapy; imaging showed continued regression of the pulmonary nodules (Fig. 2, sections $C$ and D) and his symptoms eventually resolved completely. After 12 months of docetaxel, the patient had developed grade 1 peripheral neuropathy and it was decided to allow the patient a break from chemotherapy.

Chemotherapy was held off for 9 months and repeat imaging demonstrated worsening of the lung metastases along with new metastatic lesions in the liver. It was decided to rechallenge the patient with docetaxel at a slightly reduced dose due to residual peripheral neuropathy. The patient tolerated this treatment well for 3 months with stable disease, however he unfortunately developed progressive 
disease shortly thereafter, as illustrated by follow-up CT scans. His performance status had also worsened and it was decided to halt all further systemic therapy and focus on comfort measures. The patient passed away several weeks later.

\section{Etiology}

Endometrioid ductal carcinoma is a rare histological subtype of prostate cancer, accounting for $0.4 \%$ to $0.8 \%$ of all prostate cancer cases. ${ }^{3}$ Originally, ductal carcinoma of the prostate was thought to have arisen from the prostatic utricle in the form of a mullerian ductal structure; however, immunohistochemistry studies have since shown that this rare form of prostate cancer arises from the prostatic ducts themselves. ${ }^{4}$

Ductal carcinoma of the prostate occurs most often in men between the ages of 60 and 80 , and typically presents with symptoms of urinary obstruction or hematuria due to a prostatic urethral mass. It may also arise in peripheral or secondary periurethral ducts. Given that ductal prostate cancer tends to occur near the urethra, digital rectal examination (DRE) is often normal in these patients. Referral to a urologist usually follows for staging and further workup.

Typical biopsy findings include a number of different architectural patterns including papillary, cribriform, solid and glandular structures, although many of these can coexist within the same biopsy specimen. Ductal carcinoma of the prostate is usually assigned a Gleason score of $8(4+4)$, due to the morphology and the more aggressive behaviour of the tumour. ${ }^{5}$ Although PSA is expressed by ductal carcinoma cells, serum PSA is normal in many of these patients. ${ }^{1}$ Some studies have reported on the secretion of carcinoembryonic antigen by prostate ductal carcinoma, however there do not appear to be any reliable serum tumour markers for which the extent of disease can be followed. ${ }^{6}$

\section{Discussion}

Following tissue diagnosis, most patients proceed to radical prostatectomy. Ductal prostate carcinoma is often diagnosed at a later stage than usual prostate adenocarcinoma due to the negative findings on routine DRE and normal PSA. Unfortunately, many patients with ductal prostate cancer are found to have extra-capsular extension with positive margins following surgical resection. ${ }^{7}$

Some patients with ductal carcinoma of the prostate undergo a course of external beam radiation for local disease control following surgery. Although specific guidelines do not exist as to the exact dose and scheduling of radiation therapy for ductal prostate cancer, previous cases have reported on the administration of 7000 to 8000 cGy over 30 to 40 fractions to the pelvis and regional lymph nodes, resulting in long-term local disease control. ${ }^{1,8}$ Some

\begin{tabular}{lc}
$\begin{array}{l}\text { Table 1. Prostate-specific antigen values over the } \\
\text { course of the patient's treatment }\end{array}$ \\
\hline Date & $<$ ( $\boldsymbol{\mu g} / \mathbf{L})$ \\
\hline May 2001 & 0.1 \\
\hline March 2002 & $<0.1$ \\
\hline September 2003 & $<0.1$ \\
\hline August 2004 & 0.14 \\
\hline January 2005 & 0.21 \\
\hline May 2005 & 0.31 \\
\hline November 2005 & 0.28 \\
\hline January 2006 & 0.96 \\
\hline June 2006 & 1.6 \\
\hline October 2006 & 0.04 \\
\hline February 2007 & $<0.03$ \\
\hline March 2007 & $<0.03$ \\
\hline August 2008 & $<0.03$ \\
\hline January 2009 & 0.10 \\
\hline March 2009 & 0.17 \\
\hline May 2009 & \\
\hline PSA prostate-specific antigen. & \\
\hline
\end{tabular}

case series have suggested that the extent of local disease can be significantly underappreciated in patients with ductal prostate cancer, with some patients having significant pelvic lymph node involvement, seminal vesicle invasion and extracapsular extension. ${ }^{7,9}$ For this reason, adjuvant radiation therapy following surgery is thought to be of benefit. These patients also benefit from androgen deprivation therapy (ADT), given that ductal carcinoma of the prostate tends to be hormone sensitive.

Prostate ductal carcinoma has a propensity to metastasize to the penis, testis, bone, liver, lungs and brain. ${ }^{1,2}$ Unlike most adenocarcinomas of the prostate seen commonly in clinical practice, a large case series suggests that lung and liver deposits may be present in as many as half of all patients with metastatic ductal carcinoma of the prostate. ${ }^{10}$ Metastatic ductal prostate carcinoma poses a unique challenge to urologists and oncologists in diagnosis and treatment due to its aggressive behaviour and variety in recurrence patterns. Biopsies from metastatic sites of disease can appear identical, similar or quite different on histological examination from a morphologic perspective, when compared with the primary tumour. Cytological and structural features of the metastatic lesions can appear very similar on pathology to 


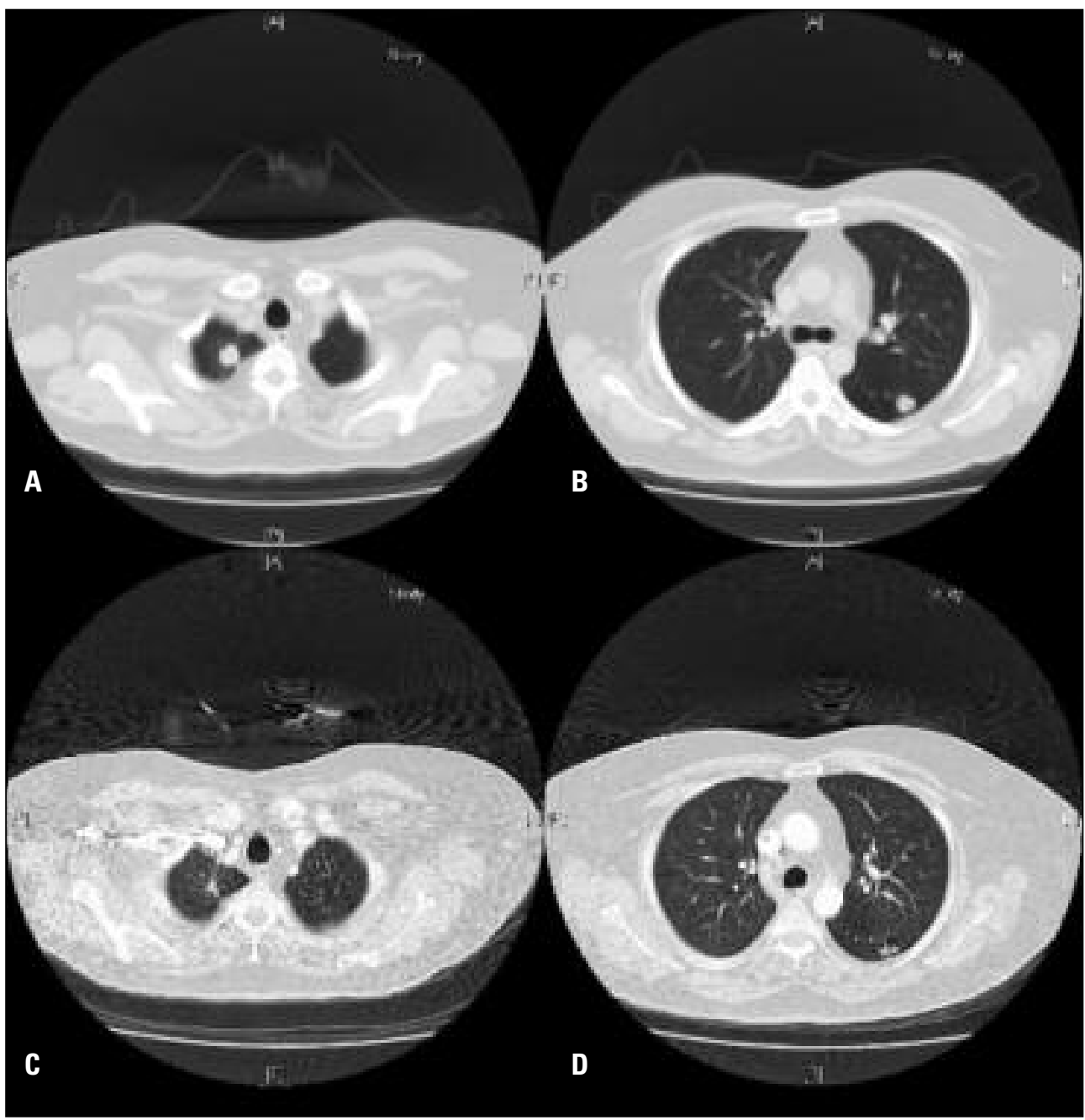

Fig. 2. Computer tomography images of the lungs. (A) February 2007, apex of the lungs, revealing lesion in right lobe. (B) February 2007, lesion in left lung, posterior segment. (C) September 2008, same lesion as in (A) now decreased in size. (D) September 2008, same lesion as in (B), now decreased in size and resolving.

other primary adenocarcinomas, such as those of the gastrointestinal tract, lung and bladder. Should metastases be identified, primary cancers from these other sites may need to be excluded when there is a clinical suspicion of other coexisting primary malignancies. ${ }^{11}$ In this case, the patient's lung and liver lesions were in keeping with metastatic ductal adenocarcinoma of the prostate based on the overall clinical picture.

Most patients with metastatic disease will initially receive ADT, which can be achieved either by bilateral orchiectomy or via a $\mathrm{GnRH}$ agonist with an anti-androgen compound. For patients with painful bone metastases, local radiation therapy can be considered. Should the ductal prostate adenocarcinoma become hormone refractory with progression of disease, visceral metastases or clinically significant symptoms from their metastases, systemic chemotherapy can be discussed with the patient. The preferred agent of choice in patients with usual metastatic hormone refrac- tory prostate cancer is intravenous docetaxel, typically administered every 3 weeks in combination with oral prednisone $5 \mathrm{mg}$ twice daily. The use of this regimen is based on the TAX-327 trial, which was the first randomized controlled trial to show a survival benefit using a cytotoxic agent for metastatic prostate cancer. ${ }^{12}$ It has yet to be determined if docetaxel is the only agent that will result in clinical tumour responses in patients with metastatic ductal carcinoma of the prostate.

\section{Conclusion}

Ductal adenocarcinoma of the prostate is a rare variant of prostate neoplasms. Guidelines and methods on how to manage and treat this disease do not exist, and current treatment is inferred based upon the management of metastatic prostate adenocarcinoma of any histological subtype.

This rare histological subtype of prostate cancer tends to 
present later than the more common variant of prostate cancer given that their serum PSA and DRE tend to be normal. In addition, many of these patients will have positive resection margins with infiltration of seminal vesicles and lymph node involvement following radical prostatectomy. Therefore, many patients receive local radiation as well as ADT following surgery.

Monitoring for recurrence can be difficult given that serum PSA values do not tend to be elevated in many patients with ductal adenocarcinoma of the prostate. Unfortunately, some patients will develop metastatic disease. In such cases, secondary hormonal manipulations can be attempted followed by systemic chemotherapy, comprising of docetaxel and prednisone, given every 3 weeks.

To the best of our knowledge, this is the first report of a patient with metastatic ductal adenocarcinoma of the prostate with a documented clinical tumour response to docetaxelbased chemotherapy. It is currently unknown as to whether docetaxel is the best agent of choice for ductal prostate cancer. What is clear though is that this variant of prostate cancer can respond to systemic chemotherapy. Moreover, experience and research in patients with ductal prostate cancer should help to determine whether some of the newer agents currently in clinical trials would be effective against this particular histological subtype of prostate cancer.

${ }^{*}$ Internal Medicine Resident, Post-Graduate Year 2, McMaster University, Hamilton, ON; ${ }^{\dagger}$ Medical Oncologist, Juravinski Cancer Centre, Hamilton, ON

Competing interests: None declared.

This paper has been peer-reviewed.

\section{References}

1. Eade TN, Tahseen A, Horwitz EM, et al. Role of Radiotherapy in Ductal (Endometrioid) Carcinoma of the Prostate. Cancer 2007;109:2011-5.

2. Copeland JN, Amin MB, Humphrey PA, et al. The morphologic spectrum of metastatic prostatic adenocarcinoma to the lung: special emphasis on histologic features overlapping with other pulmonary neoplasms. Am J Clin Path 2002;1 17:552-7.

3. Orihuela E, Green JM. Ductal prostate cancer: Contemporary management and outcomes. Urol Oncol 2008;26:368-71.

4. Botswick DG, Kindrachuk RW, Rouse RV. Prostatic adenocarcinoma with endometrioid features. Clinical, pathologic and ultrastructural findings. Am I Surg Pathol 1985;9:595-609.

5. Shazer RL, Luthringer D, Agus DB, et al. Ductal Adenocarcinoma of the Prostate. Clin Adv Hematol Oncol 2004;2:393-5.

6. Tu SM, Reyes A, Maa A, et al. Prostate carcinoma with testicular or penile metastases. Clinical, pathologic and immunohistochemical features. Cancer 2002;94:2610-7.

7. Christensen WN, Steinberg G, Walsh PC, et al. Prostatic duct adenocarcinoma. Findings at radical prostatectomy. Cancer 1991;67:2118-24.

8. Tu WH, Jensen $\mathrm{K}$, Freiha $\mathrm{F}$, et al. A case of prostatic adenocarcinoma recurrence presenting as ductal carcinoma of the prostate. Nat Clin Pract Urol 2008;5:55-8.

9. Brinker DA, Potter SR, Epstein JI. Ductal adenocarcinoma of the prostate diagnosed on needle biopsy: correlation with clinical and radical prostatectomy findings and progression. Am I Sug Pathol 1999;23:1471-9.

10. Tu S, Lopez A, Leibovici D, et al. Ductal adenocarcinoma of the prostate: Clinical features and implications after local therapy. Cancer 2009;115:2872-80.

11. Gong Y, Caraway N, Stewart J, et al. Metastatic Ductal Adenocarcinoma of the Prostate. Cytologic Features and Clinical Findings. Anat Pathol 2006;126:302-9.

12. Tannock IF, Wit R, Berry WR, et al. Docetaxel plus prednisone or mitoxantrone plus prednisone for advanced prostate cancer. N Engl J Med 2004;351:1502-12.

Correspondence: Dr. Aalok Kumar, 601 Yorkminster Cr., Mississauga, ON L5R 2Al; aalok.kumar@medportal.ca 